\title{
Antigenic Comparison of HIV Envelope Complexes Containing Either sCD4, Human Anti-envelope Monoclonal Antibody A32, or CD4 Mimic Protein CD4M9 Sani Ibrahim ${ }^{1,2}$, Kathryn Bobb ${ }^{3}$, Ilia Harris 3 , James Robinson ${ }^{4}$, Anthony DeVico ${ }^{1}$ and Timothy R Fouts* ${ }^{*}$
}

Address: ${ }^{1}$ Institute for Human Virology, Nigeria, ${ }^{2}$ Ahmadu Bello University, Nigeria, ${ }^{3}$ Profectus BioScience, Nigeria and ${ }^{4}$ Tulane University, Nigeria

Email: Timothy R Fouts* - fouts@profectusbiosciences.com

* Corresponding author ‡Presenting author

from 2005 International Meeting of The Institute of Human Virology

Baltimore, USA, 29 August - 2 September 2005

Published: 8 December 2005

Retrovirology 2005, 2(Suppl I):S69 doi:10.1I86/I742-4690-2-SI-S69

There is growing interest in using antigens that replicate the envelope transition state structures that occur during HIV entry as vaccine immunogens. Three such immunogens have been developed - complexes between gp120 and sCD4 (CD4/gp120), gp120 and a human monoclonal antibody A32 (A32/gp120), and gp120 and a CD4 mimic molecule CD4M9, SCBaL/M9. Antigenic comparisons of these immunogens revealed key differences between these complexes. Coreceptor binding is 3-fold higher with the gp120/sCD4 over gp120/A32 and gp120/ CD4M9 complexes. However, the CD4 induced epitopes (CD4i) recognized by $17 \mathrm{~b}$ and FabX5 are expressed equally between all three complexes. 19e, which recognizes an epitope that is completely dependent upon CD4 binding (CD4d), binds to gp120/sCD4 but not to A32/ gp120 or SCBaL/M9 complexes. Another CD4d epitope recognized by ED47 is similarly prominent in CD4/gp120 complexes but significant less so in A32/gp120 and $\mathrm{SCBaL} / \mathrm{M} 9$. These data indicate that the antigenic features of the A32/gp120 and SCBaL/M9 are more consistent with a transition structure between unligated gp120 and the CD4/gp120. Chemical crosslinking can obscure these $\mathrm{CD} 4 \mathrm{i}$ and $\mathrm{CD} 4 \mathrm{~d}$ epitopes. These antigenic differences may also explain the differences in the neutralizing antibody profiles generated by CD4/gp120 and A32/gp120 complexes in animal experiments. 